\title{
Gender Differences in Peer Support in Type 2 Diabetes Self-Management
} Across the Globe

\author{
Florence O. Okoro ${ }^{1^{*}}$, Debra J. Barksdale ${ }^{2}$ and Edwin B. Fisher ${ }^{3,4}$
}

${ }^{1}$ School of Nursing, University of North Carolina, 9201 University City Blvd, Charlotte, NC 28223-0001, USA

${ }^{2}$ School of Nursing, Virginia Commonwealth University, Sadie Heath Cabaniss Hall, 1100 East Leigh Street,Richmond, VA 23298-0567, USA

${ }^{3}$ Peers for Progress, Gillings School of Global Public Health, University of North Carolina, Box 7440, Chapel Hill, NC 27599-7440, USA

${ }^{4}$ Department of Health Behavior, Gillings School of Global Public Health, University of North Carolina, Box 7440, Chapel Hill, NC 27599-7440, USA

\begin{abstract}
Objective: The effectiveness of peer support programs as a means to sustain self-management in patients with type 2 diabetes has been well documented. However, little is known of how gender differences in peer support might influence the provision and receipt of support. This study used the key functions of peer support as a framework to explore gender differences in peer support programs and identified gender-related issues and global cultural contexts that influence peer support.
\end{abstract}

Methods: Qualitative data were generated through telephone interviews and written responses using open-ended questions to nine programs across the globe: Africa (Cameroon and Uganda), Asia (Cambodia, Hong Kong, Thailand, and Vietnam), and the United States (Alabama, California, and Illinois). Qualitative content analysis was done to achieve in-depth exploration of categories. Data were analyzed using INVIVO 10 software.

Results: Five themes emerged: 1) Differences in emotional support: female peer supporters reportedly displayed more emotional support than any other form of support; 2) Differences in instrumental support: male peer supporters gave information as their primary form of support; 3 ) Who is a peer supporter: males dominated as peer supporters in two Asian countries (Cambodia and Hong Kong) and females dominated in African American and Latino peer support programs in the US; 4) Matching by gender: male peer supporters were assigned only male recipients, but female peer supporters could be assigned to either sex; 5) Gender differences in participant availability: there was considerable variety in how gender was manifest in programs and both peer supporters' and participants' responses to them.

Conclusion: This study provided evidence that substantial gender differences and gender-related issues were present in all the peer support programs and that the manifestation of differences varied considerably across settings and cultures. Gender differences have to be taken into consideration when planning peer support programs.

\section{Introduction}

Diabetes is a global public health concern and has quickly grown from 366 million people suffering from the disease in 2011 to 415 million in 2015. These figures are projected to increase tremendously to 592 million people by 2035 [1-3]. To curb this epidemic, diabetes care self-management, along with disease treatment by health professionals, is critical. However, with the rapid increase in the number of people with diabetes, the available health care professionals are insufficient to provide the individualized attention that patients require [4-5]. Moreover, the cost of providing care may be beyond the resources of the health care systems of many middle and low-income countries facing the escalating number of adults with type 2 diabetes [6].

Peer-delivered support is one solution that can help with diabetes management. Peer support can be an efficient and cost-effective means assisting with self-management in patients with chronic diseases, such as diabetes [7-8]. Individualized one-on-one attention is a common feature of peer support interventions for type 2 diabetes selfmanagement $[9,5,10]$. Peer support programs have been established in many parts of the world in countries such as United States, Cambodia, China, Thailand, Vietnam, Cameroon, Uganda. These programs are considered low cost, effective, culturally acceptable, and provide long term sustained support $[8,11,10,12-14]$.

\section{Publication History:}

Received: April 08, 2017

Accepted: May 29, 2017

Published: May 31, 2017

\section{Keywords:}

Type 2 diabetes, Peer support, Social support, Self-management, Gender, Culture, Qualitative Interviews.

\section{Peer Support in Diabetes Self-Management}

Peer support is broadly defined as social support provided by someone who belongs to the same age, disease, or social group, etc. and someone of equal standing. Peer support for diabetes self-management is often delivered through organized peer support programs. The World Health Organization (WHO) consultative committee on peer support programs in diabetes endorsed peer support as an effective means of health promotion and diabetes management [15]. Peers for Progress (PfP), a global initiative based at the University of North Carolina-Chapel Hill, was developed in response to the promise of peer support as an effective means of sustaining diabetes selfmanagement. PfP leads and encourages further research to set up 'best practices' in peer support around the globe [4]. There is need for more research to achieve the goal of 'best practices' in peer support especially since not all peer support interventions yielded the desired physiological outcome [16].

"Corresponding Author: Dr. Florence O. Okoro, School of Nursing, University of North Carolina, 9201 University City Blvd, Charlotte, NC 28223, USA; E-mail: fokoro1@uncc.edu

Citation: Okoro FO, Barksdale DJ, Fisher EB (2017) Gender Differences in Peer Support in Type 2 Diabetes Self-Management Across the Globe. Int J Nurs Clin Pract 4: 239. doi: https://doi.org/10.15344/2394-4978/2017/239

Copyright: (๑) 2017 Okoro et al. This is an open-access article distributed under the terms of the Creative Commons Attribution License, which permits unrestricted use, distribution, and reproduction in any medium, provided the original author and source are credited. 


\section{Characteristics of the Peer Supporter}

Naturally, peer supporters are a crucial and important component of peer support programs. They are often recruited from the target community and receive training to enhance their ability to function as peer supporters. In the context of self-management of diabetes, a peer is someone who has diabetes, has experience with diabetes self-management via a close relative or friend with the disease or is a person who has cared for someone who has diabetes [17]. The similarity with the person being supported is one of the most important characteristics of the peer supporter. Peer support is a type of social support, which is strongly influenced by cultural factors. The peer supporter should understand the culture of the community, speak the same language as the people being supported and have similar cultural preferences. Peer support programs are designed to be culturally congruent to enhance their acceptability and success $[18$, 13].

\section{Gender and Peer Support}

Gender exerts an important influence on social support [19-21]. Gender is another area that is critically important in peer support as gender roles are influenced by culture and society. Gender roles are those socially constructed activities, characteristics, and behaviors that a given society or community generally regards as appropriate for the male or female. Gender has a great impact because some peers prefer to be matched with someone of the same gender because they may feel more comfortable discussing intimate issues of sexual problems and other role-related issues [22,11]. Gender has received little attention in peer support programs and research despite general recognition that peer support is influenced by the cultural and social dimension of the society in which it occurs. The literature has shown that there are differences in how men and women comprehend and incorporate support into their lives [19, 23, 20,21], and this may be influenced by traditional gender role patterns and socializations [21]. A better understanding of the potential differences between men and women in the engagement and response to peer support activities may help to maximize the positive effects of peer support programs in improving diabetes outcome.

Towards this end, one qualitative study that reviewed 16 peer programs in the United States reported that peer support program managers were concerned about gender and culture issues, particularly among Hispanic groups. Specifically, female peer supporters had difficulty speaking to male support recipients about problems of sexual dysfunction as a probable effect of diabetes [22]. Despite these findings, gender issues have not been addressed in major training manuals, toolkits or peer support program guidelines. Peer support training manuals accessed through the website of Peers for Progress illustrate this point [23]. For example, the Australasian Peers for Progress Diabetes Project peer leader training manual [24], the CDC Community Health Worker training manual [25], the ENCOURAGE Peer Adviser training manual [26] and the International Diabetic Federation [IDF] Peer Leader Manual [27] were reviewed and none was found to address gender issues in training peer supporters. Neither, the Peers for Progress Comprehensive Program Development Guide [28] nor the US Department of Health and Human Services document known as the Community Health Worker Evidence-Based Models toolbox [29] address gender differences in peer support provision.

Therefore, this study was undertaken to address the gap in the literature around gender issues on peer support because of its potential effect on the outcomes of peer support interventions in diabetes self-management. The purpose of this study was to explore gender differences in the services provided in peer support for Type 2 diabetes self-management with an aim to determine if and how gender is considered in planning, with the training of peer supporters and in setting up the peer support program. The study also explored the global cultural context of the male and female provision of peer support services.

\section{Study Framework: Peers for Progress Four Functions of Peer Support}

Guided by the WHO 2008 consultative committee report on peer support programs in diabetes and peer support investigators, Peers for Progress identified four key functions of peer support that can be applied globally [23]. These four key functions were the framework that guided this study.

\section{Assistance in Daily Disease Management}

Peer supporters provide services that assist people living with diabetes to manage their daily self-care. These services include 1) providing the patients information on diabetes, 2) educating patients on diabetes self-care skills, 3 ) reinforcing the education provided by the healthcare professionals, 4) identifying key resources, such as where to buy healthy foods or pleasant and convenient locations for exercise 5) assisting or accompanying patients to grocery shopping 6) assisting with blood glucose checks 7) assisting with meal preparations. Peer support programs may operationalize assistance in daily disease management by teaching individuals portion sizing, accompanying them to grocery shopping, showing them how to read food labels, healthy eating, and exercise [4]. Peer supporters use their experiences in helping people figure out how to manage diabetes in their daily lives.

\section{Social and Emotional Support}

The peer supporters provide participants opportunities to discuss personal problems either in the group or one-on-one settings. Through empathic listening, offering advice without being judgmental, and by simply being available, the peer supporters encourage their peers to stay motivated to reach their self-care goals. The peer supporters share experiences of what worked for them when they faced difficult and challenging situations in their self-care. Kowitt et al. [30], in a study on emotional support for diabetes management, reported that peer supporters described emotional support as "just being there" for peers.

\section{Linkage to Clinic Care and Community Resources}

People living with chronic disease conditions recognize that the healthcare system is complex and they may express difficulty navigating the system. Peer support programs function to help the people living with chronic disease to navigate the healthcare systems, link them to clinic care, and help them to access community resources. Also, peer support programs provide encouragement for patients to keep their clinic appointments through diverse means such as automated telephone reminders, provision of transportation, and accompanying the patients to clinic visits if needed. Peer support programs might play the role of advocates, representing the interests of the patients with government agencies, non-governmental organizations, donor agencies, philanthropists, and community groups [20]. 


\section{Ongoing Support}

Providing long-term flexible and continual follow-up keeps patients engaged in their self-care. Ongoing, sustained support helps the people with chronic disease such as diabetes to maintain self-care, avoid disease complications, and live a good quality life. Ongoing follow up support is accomplished by providing proactive communication like calling peers to remind them of their clinic appointments, maintaining personal connections with the peers, motivating and encouraging peers to continue their life long selfmanagement, and being consistent in sending key information pertinent to diabetes self-management [31]. Ongoing support can be provided through telephone calls, automated phone messages, text messages, and home visits.

\section{Method}

\section{Design}

A qualitative descriptive approach and content analysis of the responses to open-ended interview questions were used to conduct the study. Nine peer support programs for Type 2 diabetes around the world were purposefully sampled. At each site, one program manager/ key informant served as the research participant. Interview questions were organized to capture gender differences in key functions of peer support $[4,32]$ and the cultural context of the male and female provision of support but allowed flexibility to capture the perspectives of the informants. The thick description was achieved using, for the most part, words of the informants. Approval for the study was obtained from the Institutional Review Board of the University of North Carolina at Chapel Hill. Individual consent was obtained from all the research participants through the leader of Peers for Progress.

\section{Programs and participants}

The peer support programs were contacted through the leader of Peers for Progress. The nine programs contacted were located in three continents and seven countries namely: United States (Alabama, Chicago, and California); Asia (Cambodia, Hong Kong, Thailand, and Vietnam); and Africa (Cameroon and Uganda). The program manager/key informant participants chosen were based on their experience training in and working with peer supporters in their program. Details of the nine programs can be found in Table 1(Supplementary File).

\section{Data collection instrument}

The authors and a team of experts familiar with the peer support literature developed the interview questions. A structured interview guide containing ten open-ended questions was based on the four key functions of peer support that Peers for Progress has promoted to provide a standardized but adaptable template for global promotion of peer support [4]. These key functions are 1) assistance with daily disease management, 2) emotional and social support, 3) linkage to clinical care and community resources, and 4) ongoing support. See the interview questions in Table 2 (Supplementary File). Data collection spanned from October 2014 to March 2015.

The team of peer support experts from Peers for Progress reviewed the first three responses from informants to ensure that the questions were eliciting pertinent responses. The peer support experts determined that there was no need to revise the questions and, hence, the data collection proceeded. Data saturation was reached when the data generated from the responses of the nine peer support programs did not show evidence of any new themes.

\section{Data analysis}

Eight of the nine key informants who participated in the study provided written responses to the questions in English since most participants were more comfortable with written English than with English spoken over the telephone. Language distortion can occur over the telephone making comprehension, understanding, and response more difficult for the interviewer and non-native English speaking key informant, in particular. Follow up emails were used for clarifications of unclear responses and to ask further probing questions. All the emails were analyzed as data. One participant preferred a telephone interview. The interview lasted 35 minutes and was audio taped and transcribed. All the written responses, including the emails and the interview transcripts, were imported into INVIVO 10 software. Data were deductively coded based on predetermined codes generated from the literature on gender and the standardized definitions of the four key functions of peer support. Two members of the Peers for Progress research group who are experienced in qualitative research methods reviewed the coding for consistency. Weekly meetings were held until all the coding and interpretations were agreed on, and discrepancies were resolved by consensus.

\section{Results}

Nine peer support programs around the globe participated in the study. The program manager or key informant from each program responded to the interview questions. The program managers/key informants were adults $>21$ years of age, six females and three males; all respondents had at least a college degree. Five themes emerged from the data analysis. Quotations were used to illustrate the themes [33]. See Table 3 (Supplementary File) for details.

\section{Differences in Emotional Support}

Peer support program managers/key informants in eight of the nine programs reported that female peer supporters provided social and emotional support to support recipients more than any other form of support. For example, when asked to describe the support provided by male and female peer supporters or differences in the support they provided, the key informant from one program said, "But what I would observe when they would be on the phone is that I think they might have offered more social and emotional support." Another program manager said, "Female peer leaders provided the assistance with social and emotional support better than male peer leaders." A third key informant said, "the female peer supporters showed a greater sense of empathy probably due to the mother and caretaker role which they already occupied in normal day-to-day life." A fourth peer support program manager's response to the same question was, "I would say social and emotional support, female peer educators provide in general a softer voice to educate other members in their peer group" and a fifth program key informant said, "female supporters might be more capable of providing emotional support, because they are more emotional themselves."

\section{Differences in Instrumental Support}

The peer support program managers/ key informants reported that males engaged more in information sharing as their way of providing support for assistance with daily disease management. As some key informants put it, "From my experience, the male supporters (middle 
age) are more proactive and confidence in giving information to peers." Males were said to be consistent in information sharing as a key informant stated, "In my project, it seems male supporters are more engaged and consistent in information sharing." Another key informant said that males engaged more in information sharing about exercise especially the "hard exercise" but females engaged more in sharing resources inform of giving out pamphlets with this quote, "If the topic is related to hard exercise like jogging male are givers more than female, they mostly share knowledge for others but not prepare or provide any material for the others."

The two peer support programs in this study that served Latinos in the United States gave different reports on male peer supporters' engagement with daily disease management assistance. While one Latino program manager said that the males were more engaged than the females in information sharing, the other reported that there was no observed difference in how male and female peer supporters engaged in giving their peers tangible assistance. The quotes below illustrated the different points of view. Program Manager 1 -"Male participants [i.e., peer supporters] are much more straightforward with diabetes strategies (e.g., go to the doctor, take your medicines, eat right, and exercise)" and "Males didn't spend as much time as females in establishing rapport. They would get into sharing information more quickly- they wanted to offer something concrete like information." Program Manager 2 - "I don't think there were gender differences specific to helping them with their daily disease management with regards to checking your glucose, understanding your glucose results, understanding side effects of insulin, what they needed to eat/not eat, portion sizes."

\section{Who is a Peer Supporter?}

The three peer support programs in the United States reported that their peer supporters were mostly females and they struggled with recruiting male peer supporters. The key informant in one of the programs said, "More women than men on staff at our clinics, 27 of 28 peer supporters were women." Another key informant said, "We only had one peer supporter that was a male out of the group of eight or nine. I think that the end we might have lost one or two people. So it was really one, and the rest were women peer supporters." The key informant concluded by saying, "We have not had any success recruiting a male peer supporter/Community Health Worker. I know other programs have had some limited success."

Of the three peer support programs from the United States, two served Latino communities, and one served an African American community. In those serving Latino groups, both peer supporters and support recipients were predominantly females because, according to one program manager, women in that culture utilize health care services more than men. The manager said, "Hispanic women tend to seek out health services much more readily than men do; that is something that I've observed, and I've heard others also communicate that in their observations." The key informant added that "more women than men see themselves as the primary caregivers in this community and therefore they are more likely to offer support to others." The program informant also said about Latino males, "I think traditional gender roles where men are -- I think maybe seeking out health services may be seen as a weakness and men are not supposed to appear weak."

Conversely, in two peer support programs in Asia (Cambodia and Hong Kong), the key informants reported that they had mostly male peer supporters and it was difficult recruiting females in that role. Low-level education and traditional gender role that made women home based workers were the major reasons given for the difficulty in recruiting female peer supporters in both programs. One of the informants said, "MoPoTsyo peer support program consider the selection of new Peer Educators by providing an opportunity to female in its coverage areas. But some barriers such as family burden, low education background, and social discouragement maybe affect negatively their involvement as Peer Educator." Another key informant said this; "In my peer support project, 15 out of the 23 peer supporters are male, and two out of eight female peer supporters dropped out in the middle, only one male supporter dropped out. When they made the commitment, male supporters are more responsible."

In a peer support program in Africa (Uganda), the key informant reported that because men were more educated than women, they were more likely to be employed in the city, and therefore had to commute to work. The daily commute affected their ability to fulfill their role as peer supporters, and for that reason, several men dropped out of the program. Women peer supporters were not affected. The key informant said, "Men in our study were more likely to be engaged in work that involved travel. Men had higher education levels than women. One problem that arose was that men who had to travel were not available to participate as a peer supporter as consistently as others and several dropped out of the study for this reason."

\section{Matching Peer Supporters and Support Recipients by Gender}

Though matching by gender was the most desired combination expressed by the peer support programs, this was not always possible. Gender related matching issues presented in all the peer support programs studied. The challenge of sex matching was particularly daunting for those programs that had less or none of male peer supporters but had male support recipients or few of female peer supporters but a large number of female support recipients. The male peer supporter was assigned only male recipients, but female peer supporters could be assigned to either sex as the key informant said, "so we really made a conscious effort to give that one male peer supporter male patients. However, the female peer supporters - that didn't really come into play so much, like we weren't trying to intentionally give them all women and no men." One of the key informants in another program serving Latinos said, "If a man is a peer supporter or educator, he would have to be accompanied by a woman if visiting a female patient for the female to feel comfortable and for her spouse/partner not to get suspicious of his intentions."

In the two programs in Africa, cultural values and social class were used to explain the gender issues in matching peers. For example, in Uganda it was reported that due to culture, married men and married women do not associate closely together and therefore could not be paired as was stated by the informant: "One cultural value in Uganda is that married men and women do not interact too closely with the opposite sex beyond their spouse. We paired participants in dyads and triads and avoided male/female dyads." While in Cameroon, women were sensitive to the issues of social class as was reported by the program manager that "some women, especially those from the Northern part of the country feel rather uncomfortable to express themselves or even sit in the presence of men so it was anticipated that they would be put in a group in which they felt most comfortable." 
The same trend of issues with sex matching was reported in Cambodia. The peer support program in that country had more female (69\%) support recipients but the program struggled with recruiting female peer educators. Their peer educators were $71 \%$ males.

The challenges that occurred when the gender of peer supporters and participants did not match were illustrated by two issues reported by a key informant in one peer support program. One was that the husband of a female support recipient answered the telephone call from a male support provider and challenged the male support provider to talk about the wife's health problems with him. The male peer supporter felt uncomfortable, and he stopped calling that female. Also, a female peer supporter called her male support recipient several times, and he did not pick up her calls. She stopped calling him because she felt embarrassed to continuing calling someone who did not answer.

\section{Gender Differences in Participant Availability or Need}

In a peer support program that served low income African Americans, greater numbers of female participants were caregivers of older family members. As the key informant said, "Many of our female participants are caregivers for parents, grandparents, and others, which bring the challenge of time management in balancing all their duties and diabetes management." The key informant also reported that male participants were observed to have problems with cooking for themselves, especially if they lived alone or if there were no females in the household.

In the two African countries (Cameroon and Uganda), the key informants reported that more women than men participated in peer support programs. In one peer support program, the key informant reported that women were more health conscious and therefore, were quicker than men to notice any adverse health condition according to this quote, "women tend to use health care services more often than men so are more likely to become aware of their condition sooner than men." Further, women were more sensitive to issues of culture, and socioeconomic status and the peer support staff reportedly took this into consideration in the formation of groups. "Patients with similar social, professional or cultural affinity were put in the same group. This is relevant because women tend to be more sensitive to differences in these areas and would generally modify their behavior depending on whether they view the setting as threatening or friendly." Another peer support program manager reported that married men and women did not associate closely. Therefore, peer support participants were sex-matched as reported thus, "one cultural value is that married men and women did not interact too closely with the opposite sex beyond their spouse and so we paired participants in dyads and triads and avoided male/female dyads." The peer support program manager also reported that men were better educated than women, had better employment and might be employed far away from their homes. "One problem that arose was that men who had to travel were not available to participate as a peer supporter as consistently as others and several dropped out of the study for this reason."

In an Asian country (Cambodia), the peer support program manager reported that culturally women were home-based workers, and they were not expected to seek employment outside the home. "Many husbands do not want their wives to become a peer educator. Members of the household can be unhappy when the mother is not paying as much attention to them and is busy going around the area to care for other people. They demand the matriarch to be available $100 \%$."
In another Asian country (Hong Kong, China), traditional gender roles were said to be a barrier to women engaging in peer support. One peer support program informant stated, "Male patients are easier to motivate, while female patients always complain they have too many household duties to do, e.g. raising grandchildren, cooking for the family, etc." Also, male peer supporters were described as being more engaged. Those who made a commitment to the peer support program fulfilled their promises, unlike women who did not fulfill their commitments due to family and gender role responsibilities. As the key informant stated, "The male supporters (middle age) are more proactive and confident in talking to peers; female supporters (middle age) are more hesitant and less confident when talking to peers."

\section{Discussion}

Peer support programs included in this study were located on three continents, seven countries, and nine different settings. The responses of peer support program managers/key informants indicated that gender differences in the provision of peer support were apparent despite differences in nationality, culture, and ethnicity. Females reportedly provide social and emotional support more than other forms of support and males provided information as assistance with daily disease management more than other forms of support. However, there was considerable variety in how gender manifested in programs, and both peer supporters' and participants' responses to them. Gender seemed always to matter, but how it mattered varied considerably across settings.

\section{Gender and Emotional Support}

Four functional categories of social support have been reported in the literature:emotional, informational, appraisal and instrumental or tangible [34]. Emotional support conveys to recipients that they are loved and valued and is regarded by some as the most important form of support [35, 36]. Indeed, a recent study on emotional support for diabetes management suggested that emotional support should be included in any diabetes management intervention regardless of race/ ethnicity, culture, geographical location and socioeconomic status [30].

In this study, eight of the nine program informants from different cultures and countries reported that the majority of the female peer supporters engaged in behaviors that primarily reflected emotional support.

\section{Gender and Disease Management Support}

Assistance with daily management includes giving information and teaching problem solving skills, goal setting, and skill building [4]. Informational support is achieved by providing current relevant knowledge and counseling about a condition. Access to new knowledge helps the individual solve problems without feeling overwhelmed and allows for adjustment to the changes required by the condition [37]. Informational support also includes advice and guidance from people who have similar disease experiences, which helps to motivate the support recipient to persevere towards the desired goal [38].

The participants in this study indicated that men rendered assistance with daily disease management more than they spent time talking or showing empathy. For instance, one informant said, "Males didn't spend as much time as females in establishing rapport. They would get into sharing information more quickly-they wanted to offer something concrete like information." 


\section{Gender, Culture, and Social Environment}

According to the program managers/key informants' responses, gender related issues were present in all the nine settings studied but the specific manifestations of these issues were culturally and socially determined.

How gender may matter in different ways in different cultures is illustrated by the relative participation of females and males as peer supporters. In programs serving Latinos in the U.S., most peer supporters were women. Also in the U.S program for African Americans, participants were mainly females who are caregivers in their families, and this may have effect in their participation. SamuelHodge, Skelly, Headen, and Carter-Edwards [39] reported that in African American culture, females play the role of family care givers; mostly taking care of elderly parents and grandchildren. In two Asian countries, on the other hand, males' higher educational status and fewer family responsibilities facilitated their taking on the role of peer supporter. In contrast, the female gender roles of caring for the household and lower levels of education were barriers to females accepting the role of peer supporter.

\section{Questioning the Distinction between Emotional and Informational Support}

Writing in the field has often distinguished between emotional, informational, appraisal and instrumental or tangible support [40]. Kowitt et al. [30] studied how emotional support emerges in peer support for diabetes management in three distinct groups: rural African American women and urban Latinos in the U.S., and middle class retirees in the U.K. Peer supporters described support as often mixing both informational or instrumental and emotional support. Rather than separate categories, they appear to be separate dimensions that are often woven together. Others also have suggested that informational and tangible support have the potential to enhance emotional support through communicating caring from the support provider $[35,41]$. This may suggest a way to work with male peer supporters, helping them recognize how the information and instrumental support they provide may also convey important messages of emotional support.

\section{Implications for Practice}

This study provided valuable lessons to peer support program planning and development. Table 4 (Supplementary File) illustrates the major findings and implications for practice particularly in the training and supervision of peer supporters. Also, gender and cultural expectations that can influence the implementation of peer support functions must be understood and considered when structuring peer support programs. Thus it is important that program planners have a good knowlede of the culture of the people that the program is intended to serve. The culture of the people in support provision should be the chief determinant of the structure of the program. For example in those communities where women are home based workers, the peer support program may have people in the same geographic location come together in smaller groups. The monthly meeting location could rotate between the homes of the members to encourage more people to attend. The larger group may meet in the designated location once a quarter or as convenient for the group. It may also be beneficial to set up peer support programs in work places to serve the interests of those who must continue to work or commute to the cities for work. Such peer support groups may meet formally or informally during their break times. Peer support program planners will need to be creative in establishing diabetes self-management programs within the context of gender, culture and other factors that affect self-care management.

\section{Limitations and Strengths}

One limitation of this study is that some participants whose primary language was not English may not have been able to convey all the information they had regarding gender and peer support. A telephone or face-to-face interview conducted in each of the native laguages may have yielded more data. However, a substantial amount of information was provided through the written responses, averaging 1756 words - over five double-spaced pages- per informant when transcribed into text documents. Another limitation was that the responses of participants who were key informants for the peer support programs reflected their perspectives, which may have been influenced by their personal gender biases. However, the participants received a briefing on the need to be aware of their gender biases and the importance of not letting their biases influence their responses to the interview questions.

Nevertheless, a strength of this study was that data were collected directly from informants who had extensive experience with peer support programs. An additional strength of the study was the diverse sample of countries, cultures, and programs from which observations of the roles of gender were drawn. A key omission, of course, is the lack of data from recipients of support, which is a necessary next step to confirm the findings of this study. Issues that may need further investigation include the ways in which support recipients view support from largely female peer supporters or largely male peer supporters.

This study provides evidence that there are significant genderrelated issues, which are manifest in peer support programs. Reports from respondents revealed gender differences in all the peer support programs studied, though the manner in which gender differences were manifest varied in different settings and countries. These gender differences in peer support provision have implications for planning and development of peer support program for improved behavioral and clinical outcomes. More studies are needed to understand better, how these may be incorporated in peer support programs. The study also gives insight into the difficulties or challenges that managers of peer support programs may have in adequately addressing gender in these programs in different cultures.

\section{Competing Interests}

The authors declare that they have no competing interests.

\section{References}

1. Bloomgarden $Z$ (2016) Questioning glucose measurements used in the International Diabetes Federation (IDF) Atlas. J Diabetes 8: 746-747.

2. Guariguata L, Whiting DR, Hambleton I, Beagley J, Linnenkamp U, et al. (2014) Global estimates of diabetes prevalence for 2013 and projections for 2035. Diabetes Res Clin Pract 103: 137-149.

3. International Diabetes Federation (2015) IDF Diabetes Atlas Update poster, 7th edition. Brussels, Belgium: International Diabetes Federation.

4. Boothroyd RI, Fisher EB (2010) Peers for Progress: Promoting peer support for health around the world. Fam Prac 27: i62-i68. 
Citation: Okoro FO, Barksdale DJ, Fisher EB (2017) Gender Differences in Peer Support in Type 2 Diabetes Self-Management Across the Globe. Int J Nurs Clin Pract 4: 239. doi: https://doi.org/10.15344/2394-4978/2017/239

Page 7 of 7

5. Fisher EB, Boothroyd RI, Coufal MM, Baumann LC, Mbanya JC, et al. (2012a) Peer support for self-management of diabetes improved outcomes in international settings. Health Aff (Millwood), 31: 130-139.

6. Murray NJ, Gasper AV, Irvine L, Scarpello TJ, Sampson MJ (2012) A motivational peer support program for type 2 diabetes prevention delivered by people with type 2 diabetes: The UEA-IFG feasibility study. Diabetes Educ 38: 366-376.

7. Gillespie P, O'Shea E, Paul G, O'Dowd T, Smith SM (2012) Cost effectiveness of peer support for type 2 diabetes. Int $\mathrm{J}$ Technol Assess Health Care 28: 3-11

8. Assah FK, Atanga EN, Enoru S, Sobngwi E, Mbanya JC (2015) Community based peer support significantly improves metabolic control in people with Type 2 diabetes in Yaoundé, Cameroon. Diabet Med 32: 886-889.

9. Sacco WP, Malone JI, Morrison AD, Friedman A, Wells K (2009) Effect of a brief, regular telephone intervention by paraprofessionals for type 2 diabetes. J Behav Med 32: 349-359.

10. Chan JC, Sui Y, Oldenburg B, Zhang Y, Chung HH, Goggins, et al. (2014) Effects of telephone-based peer support in patients with type 2 diabetes mellitus receiving integrated care: a randomized clinical trial. JAMA Intern Med 174: 972-981.

11. Baumann LC, Frederick N, Betty N, Jospehine E, Agatha N (2015) A demonstration of peer support for Ugandan adults with type 2 diabetes. Int J Behav Med 22: 374-383.

12. Heisler M, Vijan S, Makki F, Piette JD (2010) Diabetes control with reciprocal peer support versus nurse care management: a randomized trial. Ann Intern Med 153. 507-515.

13. Philis-Tsimikas A, Fortmann A, Lleva-Ocana L, Walker C, Gallo LC (2011) Peer-led diabetes education programs in high-risk Mexican Americans improve glycemic control compared with standard approaches a project Dulce Promotora randomized trial. Diabetes Care 34: 1926-1931.

14. Liu Y, Han Y, Shi J, Li R, Li S, et al. (2015) Effect of peer education on Self-management and psychological status in type 2 diabetes patients with emotional disorders. J Diabetes Investig 6: 479-486.

15. World Health Organization (2008) Peer support programs in diabetes: Report of a WHO consultation 5-7 November 2007. World Health Organization, Geneva, Switzerland

16. Rotheram-Borus MJ, Tomlinson M, Gwegwe M, Comulada WS, Kaufman $\mathrm{N}$, et al. (2012). Diabetes buddies peer support through a mobile phone buddy system. Diabetes Educ 38: 357-365.

17. Heisler M, Spencer M, Forman J, Robinson C, Shultz C, et al. (2009) Participants' assessments of the effects of a community health worker intervention on their diabetes self-management and interactions with healthcare providers. Am JPrev Med 37: S270-279.

18. Haltiwanger EP, Brutus H (2012) A culturally sensitive diabetes peer support for older Mexican-Americans. Occup Ther Int 19: 67-75.

19. Cheng H, Sit JW, Chan CW, So WK, Choi KC, et al. (2013) Social support and quality of life among Chinese breast cancer survivors: Findings from a mixed methods study. Eur J Oncol Nurs, 17: 788-796.

20. Gordillo V, Fekete E, Platteau T, Antoni M, et al. (2009) Emotional support and gender in people living with HIV: Effects on psychological well-being. $J$ Behav Med 32: 523-531.

21. Matud MP, Ibáñez I, Bethencourt JM, Marrero R, Carballeira M (2003) Structural gender differences in perceived social support. Personality and Individual Differences 35: 1919-1929.

22. Cherrington A, Ayala GX, Amick $H$, Allison J, Corbie-Smith G,et al. (2008) Implementing the community health worker model within diabetes management challenges and lessons learned from programs across the United States. Diabetes Educ 34: 824-833.

23. Fuhrer R, Stansfeld SA (2002) How gender affects patterns of social relations and their impact on health: A comparison of one or multiple sources of support from "close persons". Soc Sci Med 54: 811-825.

24. Engel L. Eds: Oldenburg B and Reddy P (2010) Australasian Peers for Progress Diabetes Project PeerLeader Training Manual. Melbourne: Australian Institute of Health Policy Studies, Monash University.

25. Center for Disease Control (CDC), National Center for Chronic Disease Prevention and Promotion (2014) A Community Health Worker Training Resource for Preventing Heart Disease and Stroke.

26. Andreae S (2007) ENCOURAGE: Peer advisor training manual. University of Alabama Diabetes Research and Training Center.
27. International diabetes Federation: Www.idf.org (2012) Medicine on the Net 18: 20.

28. Fisher EB (2005) Peers for Progress program development guide: A comprehensive flexible approach. Department of Health Behavior Gillings School of Global Public Health. University of North Carolina at Chapel Hill.

29. CHW Central (2011) Community Health Workers Evidence-based Models Toolbox-HRSA Office of Rural Health Policy.

30. Kowitt SD, Urlaub D, Guzman-Corrales L, Mayer M, Ballesteros J, et al. (2015) Emotional support for diabetes management: An international cross-cultural study. Diabetes Educ 41: 291-300.

31. Fisher EB, Brownson CA, O'Toole ML, Anwuri VV (2007) Ongoing follow-up and support for chronic disease management in the Robert Wood Johnson Foundation Diabetes Initiative. Diabetes Educ 33: 201S-207S.

32. Fisher EB, Ballesteros J, Bhushan N, Coufal MM, Kowitt SD, et al (2015) Key features of peer support in chronic disease prevention and management. Health Aff (Millwood) 34: 1523-1530.

33. Elo S, Kyngäs $H(2008)$ The qualitative content analysis process. J Adv Nurs 62: 107-115.

34. Cohen S, Underwood LG, Gottlieb BH (Eds.) (2000) Social support measurement and intervention: A guide for health and social scientists. Oxford University Press.

35. Burleson BR (2003) The experience and effects of emotional support What the study of cultural and gender differences can tell us about close relationships, emotion, and interpersonal communication. Personal Relationships 10: 1-23

36. Smailhodzic E, Hooijsma W, Boonstra A, Langley DJ (2016) Social media use inhealthcare: A systematic review of effects on patients and on their relationship with healthcare professionals. BMC Health Serv Res Aug 26: $442-455$

37. Iannino-Renz R (2016) The Role of Social Support in Persons with Type 2 Diabetic Wounds. MEDSURG 25: 357-359.

38. Chiu YC, Hsieh YL (2013) Communication with fellow cancer patients: Writing to be remembered, gain strength, and find survivors. J Health Psychol 12: 1572-1581

39. Samuel-Hodge CD, Skelly AH, Headen S, Carter-Edwards L (2005) Familia Roles of Older African-American Women with Type 2 Diabetes: Testing of a New Multiple Caregiving Measure. Ethn Dis 15: 436-42.

40. Setoyama Y, Yamazaki Y, Namayama K (2011) Benefits of peer support in online Japanese breast cancer communities: differences between lurkers and posters. J Med Internet Res 13: e122.

41. Schaefer C, Coyne JC, Lazarus RS (1981) The health-related functions of social support. J Behav Med4: 381-406. 


\section{Supplementary File}

\begin{tabular}{|c|c|c|c|}
\hline Country/State/City & Focus of the program & Population & Setting \\
\hline Alabama & $\begin{array}{l}\text { Diabetes management for African Americans with } \\
\text { poorly controlled (A1c }>7.5) \text { type } 2 \text { diabetes; telephone } \\
\text { intervention supplemented with monthly support groups } \\
\text { over } 12 \text { months }\end{array}$ & $\begin{array}{l}\text { Rural low-income African } \\
\text { Americans with Type } 2 \\
\text { diabetes }\end{array}$ & $\begin{array}{l}\text { Community based/clinic. } \\
\text { Participants were recruited from } \\
\text { a local safety-net primary care } \\
\text { clinic }\end{array}$ \\
\hline California & $\begin{array}{l}\text { Peer support for diabetes self-management of Latino } \\
\text { adults with A1c levels }>7\end{array}$ & $\begin{array}{l}\text { Latino adults }>18 \text { years } \\
\text { with Type } 2 \text { diabetes }\end{array}$ & $\begin{array}{l}\text { Non-profit community clinic/ } \\
\text { Federally Qualified Health } \\
\text { Center (Clinicas de Salud del } \\
\text { Pueblo, Inc) }\end{array}$ \\
\hline Cambodia & Diabetes self-management & $\begin{array}{l}\text { Rural and urban adults } \\
\text { over } 18 \text { years with Type } 2 \\
\text { diabetes }\end{array}$ & Community based organization \\
\hline Cameroon & $\begin{array}{l}\text { Diabetes management for adults with poorly controlled } \\
\text { diabetes }(\mathrm{HbA} 1 \mathrm{c}>7)\end{array}$ & $\begin{array}{l}\text { Urban adults with Type } 2 \\
\text { diabetes }\end{array}$ & $\begin{array}{l}\text { Participants recruited from } \\
\text { hospital but peer support } \\
\text { activities within the community }\end{array}$ \\
\hline Illinois & Diabetes self-management and regular, appropriate care & $\begin{array}{l}\text { Latino adults with type } 2 \\
\text { diabetes }\end{array}$ & $\begin{array}{l}\text { Federally Qualified Health } \\
\text { Centers (FQHC) }\end{array}$ \\
\hline Hong Kong & $\begin{array}{l}\text { Frequent contacts via a telephone based peer support } \\
\text { program to improve cardiogenic risk and health outcomes } \\
\text { by enhancing psychological well-being and self-care in } \\
\text { patients }\end{array}$ & $\begin{array}{l}\text { Adult patients with Type } 2 \\
\text { diabetes }\end{array}$ & $\begin{array}{l}3 \text { publicly funded hospital-based } \\
\text { diabetes centers }\end{array}$ \\
\hline Thailand & Diabetes conversation map & $\begin{array}{l}\text { Urban adults with type } 2 \\
\text { diabetes }\end{array}$ & Hospital \\
\hline Uganda & $\begin{array}{l}\text { Peer support for adults with diabetes to test the feasibility } \\
\text { and short-term impact on perceptions of social support, } \\
\text { psychological well-being and glycemic control through } \\
\text { engaging participants in diabetes self-care behaviors and } \\
\text { fostering linkages to healthcare providers }\end{array}$ & $\begin{array}{l}\text { Adults in rural Uganda with } \\
\text { Type } 2 \text { diabetes }\end{array}$ & Rural district hospitall \\
\hline Vietnam & $\begin{array}{l}\text { Diabetes self-management support intervention for } \\
\text { adults } 30 \text { years or older with poorly controlled diabetes } \\
\text { (HBA1c }>7 \text { in most recent } 3 \text { months) }\end{array}$ & $\begin{array}{l}\text { Urban adults with Type } 2 \\
\text { diabetes }\end{array}$ & University research center \\
\hline
\end{tabular}

Table 1: Description of the Peer Support Programs.

A. Differences in Type of Support Provided By and To Female and Male Participants

1. How would you describe the support provided by male and female peer supporters or differences in the support they provide in relation to:

a. Assistance with daily disease management?

b. Emotional support?

c. Linkage to clinic care and community resources?

d. What about ongoing support?

2. In general, how did men and women differ in providing support?

3. What, if anything, affected how men and women provided support?

4. What if anything affected how men and women received or responded to support

B. Differences in Participation of Female and Male Peer Supporters

1. Over the course of the program, were there differences in how the male and female peer supporters participated?

Please describe?

2. Were there trends in participation over time?

If so, did they vary by gender?

3. What, if anything, worked in dealing with these gender differences?

Please tell me more about this?

Is there anything else you'd like to add that we haven't talked about?

Table 2: Interview questions. 


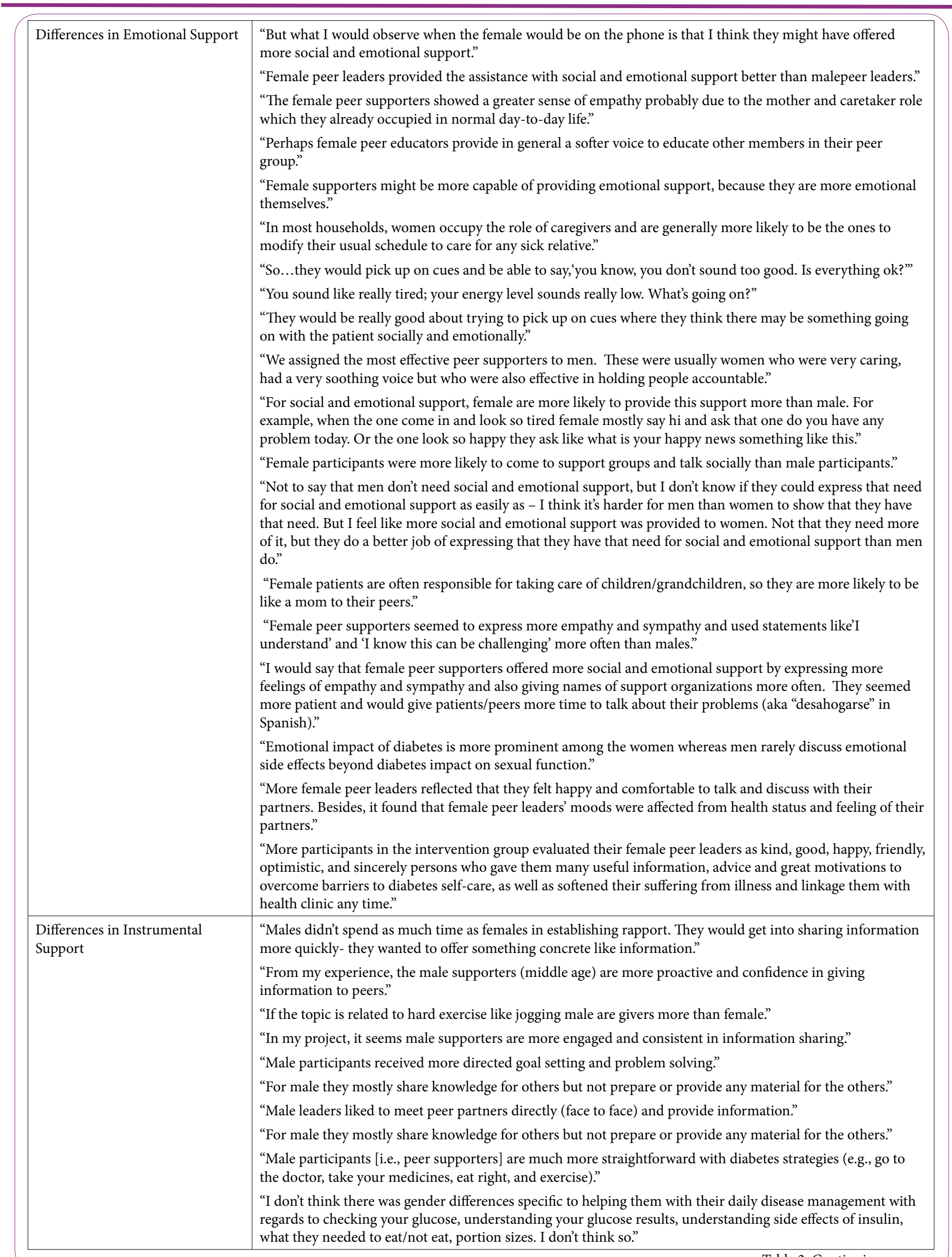

Table 3: Continuing. 


\begin{tabular}{|c|c|}
\hline Who is a Peer Supporter? & 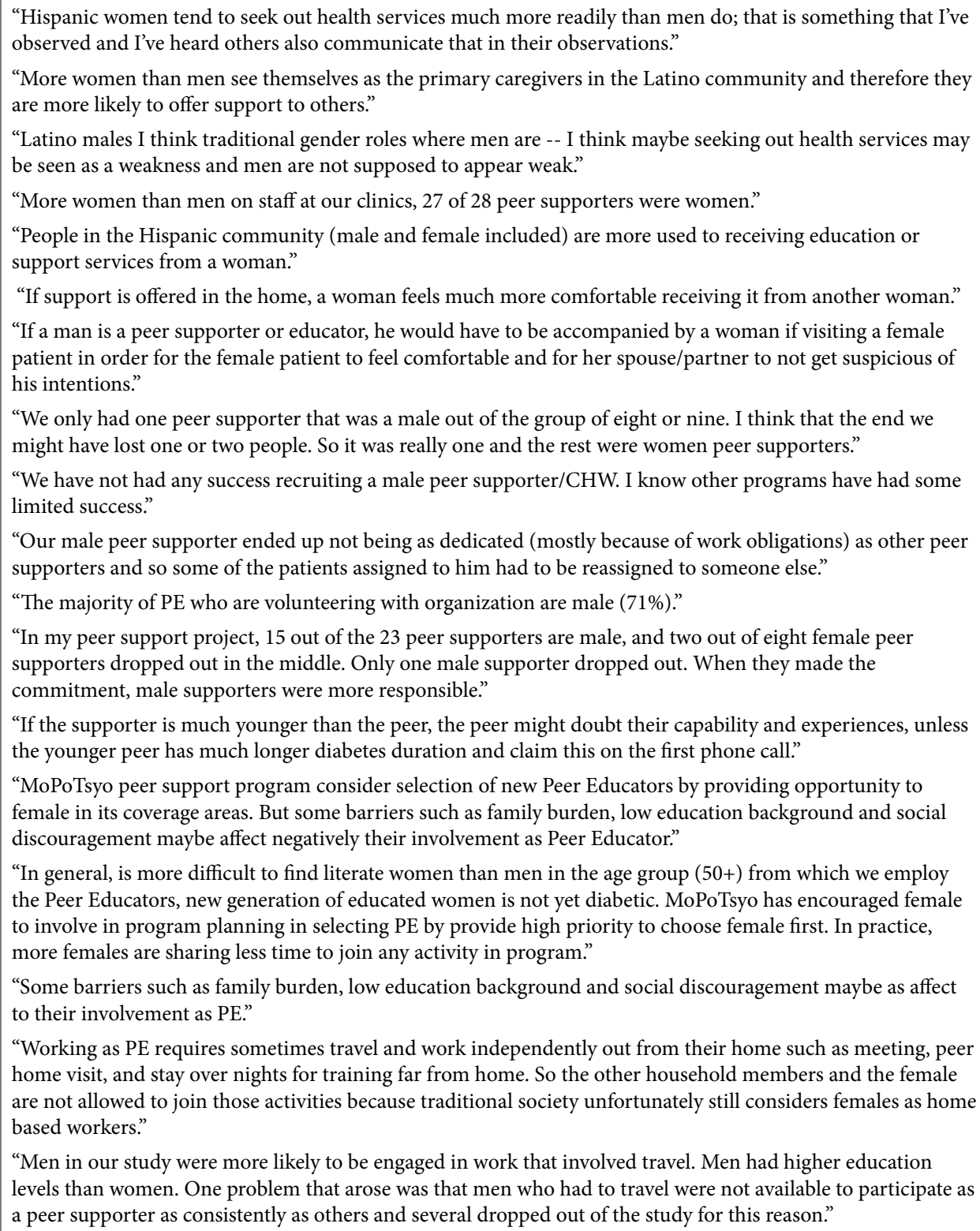 \\
\hline $\begin{array}{l}\text { Matching Peer Supporters and } \\
\text { Support Recipients by Gender }\end{array}$ & $\begin{array}{l}\text { "We paired participants in dyads and triads and avoided male/female dyads." } \\
\text { "So we really made a conscious effort to give that one male peer supporter male patients. However, the female } \\
\text { peer supporters - that didn't really come into play so much. Like we weren't trying to intentionally give them } \\
\text { all women and no men." } \\
\text { "There were times where the patients would come into the clinic and see their peer supporter one-on-one } \\
\text { since they would already be in the clinic and do whatever follow-up they needed to with that peer supporter } \\
\text { and whatnot. And so traditionally because of like very traditional gender roles and things like that - we didn't } \\
\text { want to go down that road where people would question why a female patient had a male peer supporter and } \\
\text { what's going on with that male peer supporter and why are you guys meeting in a private office, because that } \\
\text { happens". } \\
\text { "Our patients - a lot of them have immigrated into this country so bring with them, still, this very way of } \\
\text { thinking. And so - and I've seen it in other work places - we didn't want to provide any opportunity for those } \\
\text { kind of tensions to possibly happen within a family." } \\
\text { "And a lot of times, a lot of their contact with patients was over the phone so we didn't want the male partner } \\
\text { of a female patient thinking why is this person - this male person at Alivio - calling you constantly. So we } \\
\text { didn't want to offer any opportunity for that to possibly happen." } \\
\text { "We assigned only male patients to male peer supporters. For women peer supporters, patients were assigned } \\
\text { to them regardless of gender. We just tried to match them to those that lived closest to them." }\end{array}$ \\
\hline
\end{tabular}
Table 3: Continuing.. 


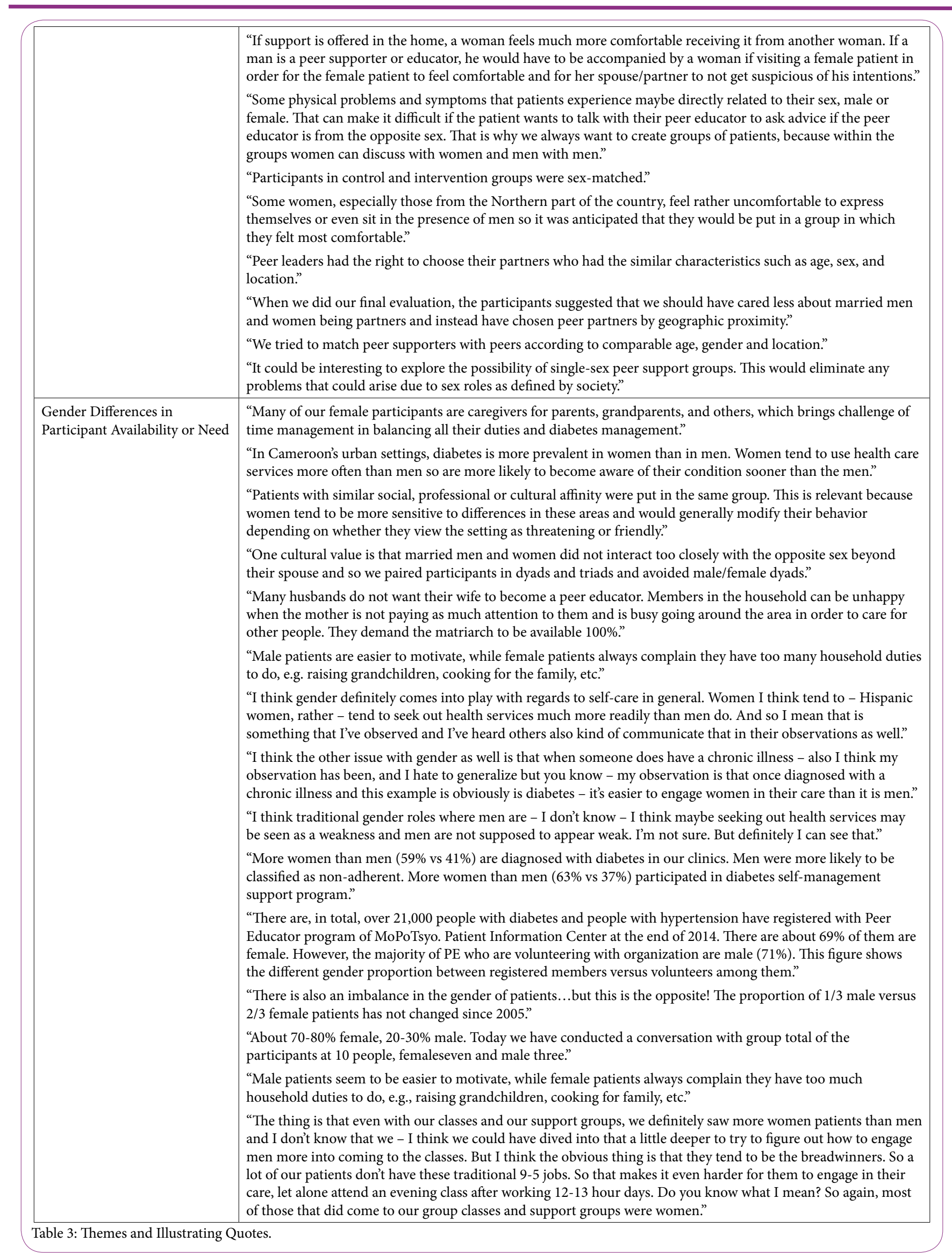


Citation: Okoro FO, Barksdale DJ, Fisher EB (2017) Gender Differences in Peer Support in Type 2 Diabetes Self-Management Across the Globe. Int J Nurs Clin Pract 4: 239. doi: https://doi.org/10.15344/2394-4978/2017/239

\begin{tabular}{|l|l|}
\hline Finding & Implications for Peer Support Programs \\
\hline $\begin{array}{l}\text { Differences in emotional } \\
\text { support } \\
\text { Differences in instrumental } \\
\text { support }\end{array}$ & $\begin{array}{l}\text { Training should emphasize importance of both emotional and instrumental/informational support as well as } \\
\text { sensitivity to women's, men's, and individuals'preferences for type of support and how it is delivered. } \\
\text { Supervision and monitoring should be constructed to avoid missing important emotional needs of clients, especially } \\
\text { when male coaches provide peer support. Likewise, instrumental tasks and objectives should be monitored more } \\
\text { closely when female peer supporters are involved. }\end{array}$ \\
\hline Who is a peer supporter? & $\begin{array}{l}\text { Understanding the likely gender distribution of the peer supporters should significantly influence training content, } \\
\text { paying attention to the aspects of support that may receive the least attention by the dominant gender peer } \\
\text { supporters. For instance, if more men are peer supporters while more women are support recipients, training should } \\
\text { emphasize those aspects of peer support that will make the participants feel well supported. }\end{array}$ \\
\hline $\begin{array}{l}\text { Matching peer supporters } \\
\text { and support recipients by } \\
\text { gender }\end{array}$ & $\begin{array}{l}\text { Gender matching of peer supporters and participants should be included in program design where practicable. } \\
\text { Gender-matched peer supporters and participants may be more comfortable providing/receiving support and } \\
\text { discussing certain personal issues, such as sexual concerns. } \\
\text { When it is not possible to gender-match peer supporters and participants, programs should have built-in systems for } \\
\text { how participants can get the emotional and informational support that they need. }\end{array}$ \\
\hline
\end{tabular}

